\section{Hsp10, Hsp70, and Hsp90 immunohistochemical levels change in ulcerative colitis after therapy}

\section{G. Tomasello, ${ }^{1}$ C. Sciumè, ${ }^{1}$ F. Rappa, ${ }^{2}$}

V. Rodolico, ${ }^{3}$ M. Zerilli, ${ }^{3}$ A. Martorana, ${ }^{3}$

G. Cicero, ${ }^{4}$ R. De Luca, ${ }^{4}$ P. Damiani, ${ }^{5}$

F.M. Accardo, ${ }^{6}$ M. Romeo, ${ }^{7}$ F. Farina, ${ }^{2}$

G. Bonaventura, ${ }^{2}$ G. Modica, ${ }^{1}$ G. Zummo, ${ }^{2}$

E. Conway de Macario, ${ }^{8}$

A.J.L. Macario, ${ }^{8,9}$ F. Cappello 2,9

'Dipartimento di Discipline Chirurgiche ed Oncologiche, Università di Palermo;

2Dipartimento di Biomedicina

Sperimentale e Neuroscienze Cliniche,

Università di Palermo, Italy;

${ }^{3}$ Dipartimento di Patologia Umana,

Università di Palermo, Italy;

${ }^{4}$ Dipartimento di Oncologia, Università di

Palermo, Italy; ${ }^{5}$ Dipartimento di Medicina

Interna e delle Malattie Emergenti,

Università di Palermo, Italy;

${ }^{6}$ Dipartimento di Contabilità Nazionale ed

Analisi dei Processi Sociali, Università di

Palermo, Italy; ${ }^{7}$ Istituto di Medicina

Biologica, Milano, Italy; ${ }^{8}$ Department of

Microbiology and Immunology, School of

Medicine, University of Maryland at

Baltimore, and IMET, Baltimore, MD,

USA; 'Istituto Euro-Mediterraneo di

Scienza e Tecnologia (IEMEST), Palermo, Italy

\section{Abstract}

Ulcerative colitis (UC) is a form of inflammatory bowel disease (IBD) characterized by damage of large bowel mucosa and frequent extra-intestinal autoimmune comorbidities. The role played in IBD pathogenesis by molecular chaperones known to interact with components of the immune system involved in inflammation is unclear. We previously demonstrated that mucosal Hsp60 decreases in UC patients treated with conventional therapies (mesalazine, probiotics), suggesting that this chaperonin could be a reliable biomarker useful for monitoring response to treatment, and that it might play a role in pathogenesis. In the present work we investigated three other heat shock protein/molecular chaperones: Hsp10, Hsp70, and Hsp90. We found that the levels of these proteins are increased in UC patients at the time of diagnosis and decrease after therapy, supporting the notion that these proteins deserve attention in the study of the mechanisms that promote the development and maintenance of IBD, and as biomarkers of this disease (e.g., to monitor response to treatment at the histological level).

\section{Introduction}

Heat shock proteins (Hsps) are a family of molecules highly conserved throughout evolution that are typically involved in folding, refolding, translocation and degradation of intracellular proteins under normal and stress conditions. ${ }^{1,2}$ In addition, Hsps have received attention from immunologists because, when present in the extracellular environment, they can trigger an inflammatory response. ${ }^{3}$ Hsps can stimulate innate and adaptive immune responses and can also, by virtue of the sequence similarity between bacterial and human orthologs, become primary targets of autoimmunity due to a phenomenon known as molecular mimicry. ${ }^{4}$ Thus, Hsps have been implicated in the pathogenesis of a number of chronic inflammatory and autoimmune diseases, like atherosclerosis, ${ }^{5}$ diabetes, ${ }^{6}$ rheumatoid arthritis, ${ }^{7}$ myasthenia gravis,${ }^{8}$ and inflammatory bowel disease (IBD). ${ }^{9}$ The latter includes Crohn's disease (CD) and ulcerative colitis (UC), both affecting the gastrointestinal tract and characterized by a chronic inflammation with mucosal damage. ${ }^{10}$ The pathogenesis of CD and UC is not clear but stress is among the suspected causative factors. ${ }^{11}$

In a previous work, we demonstrated that Hsp60 and Hsp10 are increased in the affected intestinal mucosa from patients with CD or UC. ${ }^{12}$ Further, we demonstrated that mucosal Hsp60 levels in UC patients decrease after therapy with either mesalazine alone or mesalazine plus probiotics, with the decrease in the latter combined treatment being more pronounced than in patients treated with mesalazine alone. ${ }^{13}$ All together these data indicated that determination of $\mathrm{Hsp60}$ in intestinal mucosa could be a reliable biomarker to monitor disease progression and response to treatment. The data also supported the hypothesis that Hsps, at least Hsp60, are active players in UC pathogenesis. In the present work, we measured by immunohistochemistry the mucosal levels of other Hsps (i.e., Hsp10, Hsp70, and Hsp90, all of which have been implicated in triggering/maintaining inflammation) in the same groups of patients previously studied..$^{13}$
Correspondence: Prof. Francesco Cappello, Dipartimento di Biomedicina Sperimentale e Neuroscienze Cliniche, Sezione di Anatomia Umana, via del Vespro 129, 90127, Palermo, Italy. Tel/Fax: +39.091.6553580.

E-mail: francapp@hotmail.com

Key words: ulcerative colitis, heat shock proteins, Hsp, molecular chaperones, inflammation, comorbidity.

Acknowledgements: This work was supported by Funds from the University of Palermo (VR and $\mathrm{FC}$ ) and by the Istituto Euro-Mediterraneo di Scienza e Tecnologia (FC and AJLM).

Contributions: GT, GC, GM, FF, GZ, study conception and design; GT, CS, MR, patients selection and endoscopy examination and tissue biopsy performing; VR, AM, histopathological diagnosis for ulcerative colitis performing; FR, MZ tissue preparation and immunohistochemistry performing; GB, acquisition/analysis of data substantial contribution; FMA, statistical analyses; FR, FC, FF, quantitative immunohistochemical analysis of all samples performing; RDL, $\mathrm{PD}$, data interpretation substantial contribution; FC data analysis and interpretation contribution, article drafting; ECdM, AJLM, data analysis and interpretation contribution, final manuscript writing;

GT, CS, final approval of the manuscript publishing version. GT, CS, contributed equally to the present work.

Conflict of interest: the authors report no conflicts of interest.

Received for publication: 12 September 2011. Accepted for publication: 17 October 2011.

This work is licensed under a Creative Commons Attribution NonCommercial 3.0 License (CC BYNC 3.0).

(C) Copyright G. Tomasello et al., 2011

Licensee PAGEPress, Italy

European Journal of Histochemistry 2011; 55:e38 doi:10.4081/ejh.2011.e38

\section{Materials and Methods}

\section{Patients}

We included in this study mucosal biopsies previously obtained from 40 patients with mildly to moderately active UC without systemic comorbidities, as previously described. ${ }^{13}$ Disease activity was assessed using the Mayo Score system from 0 to $12 .{ }^{14}$ Mildly to moderately disease activity was defined by a score of 4 to 10 . Biopsies were obtained at first diagnosis and after therapy. Patients were treated for 6 months with 1.2 or $2.4 \mathrm{~g} /$ die (depending on disease severity) of mesalazine (5-ASA, Asacol $^{\circledR}$, Giuliani \& Bracco, Milan, Italy) or 
with both 5-ASA and 1 capsule/die of probiotics (Acronelle $^{\circledR}$, Bromatech, Milan, Italy), as previously described. ${ }^{13}$ All the procedures followed were in accordance with the ethical standards of the responsible committees (institutional and national) on human experimentation and with the Helsinki Declaration of 1975 (as revised in 2008). Informed consents were obtained from all patients at the time of biopsy and the study was approved by our local Ethics Committee.

\section{Tissue preparations}

All biopsies were formalin-fixed and paraffin embedded. We also included in our study 20 specimens from archival tissue of formalinfixed, paraffin-embedded colon mucosa from patients with non-specific colitis, as controls. These patients underwent colonoscopy because they were suspected to suffer from intestinal inflammatory disease, but the histopathological examination excluded presence of IDB. Five $\mu \mathrm{m}$ sections were obtained from all paraffin blocks and were de-waxed and re-hydrated for immunohistochemical analysis.

\section{Immunohistochemistry}

Immunostaining was done using an avidinbiotin complex kit (LSAB2, DAKO, Carpinteria, CA, USA), as previously described..$^{15}$ Briefly, after incubation for $10 \mathrm{~min}$ with serum-free protein blocking, the following primary antibodies were added to the sections: anti-Hsp10 (dilution 1:400, Cat. No. SPA-110, StressGen Biotechnologies Co., Victoria, BC, Canada), or anti-Hsp70 (dilution 1:200, Cat. No. SC-24, Santa Cruz Biotechnology, Santa Cruz, CA, USA), or anti-Hsp90 (dilution 1:200, Cat. No. SC-13119, Santa Cruz Biotechnology). Appropriate positive controls, as well as nonimmune serum for negative controls, were run concurrently. 3-3'-diaminobenzidine (DAB chromogen solution, DAKO, Glostrup, Denmark) was used as developer chromogen. Nuclear counterstaining was done using haematoxylin (DAK0). Two independent observers (FR and FC) examined the specimens in a blind (code marked) procedure and performed a quantitative analysis to determine the percentage of cells positive for Hsps both in epithelium (Ep) and lamina propria (LP) of colon mucosa. Results were expressed in a semiquantitative scale (-: 0\%; +: 1-33\%; ++: $34-66 \%$; +++: $67-100 \%)$. All the observations were made at a magnification of $400 \mathrm{X}$ and the means of duplicate counts were used for statistical analyses.

\section{Statistical analyses}

Data obtained from immunohistochemistry evaluations were plotted using Microsoft Excel software (Microsoft Italia, Milan, Italy).
Statistical analyses were carried out using the GraphPad Prism 4.0 package (GraphPad Inc., San Diego, CA, USA). Standard statistical analyses were employed to calculate the means and the standard deviations (SD). One-way analysis of variance was used to assess significant differences within the data. Differences between the means were considered significant when a value of $\mathrm{P}<0.05$ was obtained. In order to have an indication on the variability of immunoreactivity data obtained by the different observers, the coefficient of variation was calculated using both the inter-observer and intra-observers kappa statistics.

Finally, we performed a correlation analysis between immunohistochemical levels of Hsps and of inflammatory markers, i.e., CD3, CD4, CD8, CD20, and CD68 (data that we have obtained in a previous work $^{13}$ ), using the Pearson's test. For correlation graphs, linear regression was also calculated to reflect the type of correlation between the chosen variables. Data were considered significant at an alpha-level of $5 \%$.

\section{Results}

We divided the samples in four groups: mucosa obtained at time of diagnosis (group T0); mucosa obtained after 6 months of treatment with 1.2 or $2.4 \mathrm{~g} / \mathrm{die}$ of 5-ASA (group T1a); mucosa obtained after 6 months of treatment with 5-ASA + probiotics (group T1b); mucosa obtained from patients with non-specific colitis, as control (group C). Figure 1 shows representative pictures of immunohistochemical results and the pertinent semiquantitative data are displayed in Table 1, in which the levels of the Hsps investigated are shown for the epithelium (Ep) and lamina propria (LP). Hsps levels in T0 specimens tended to be higher than in the controls $(\mathrm{C})(\mathrm{P}<0.05)$.

Hsp10 levels in Ep and LP were lower in the Tla and in Tlb groups $(\mathrm{P}<0.05)$, compared to $\mathrm{T} 0$, with the most marked decrease occurring in the T1b group (T0 vs T1b:P $<0.001$; Tla vs
T1b: $\mathrm{P}<0.005)$. The Hsp10 levels in the T1b group were comparable to those of the controls, C ( $>>0.05)$.

Hsp70 levels tended to be higher in Ep than in LP, in which its positivity was mild. Ep levels declined significantly from T0 through Tla to T1b (T0 E T1a: P<0.05; T0 CE T1b: P<0.005; T1a $\mathrm{E}$ T1b: $\mathrm{P}<0.01)$. T1b levels were comparable to those of controls, $\mathrm{C}(\mathrm{P}>0.05)$.

Hsp90 levels were also higher in Ep than in LP. However, its levels in Ep did not present any significant change among Groups T0, T1a, and T1b, while Group C had significant lower levels $(\mathrm{P}<0.01)$. Hsp90 levels in LP of T0 and T1a did not change significantly. By contrast, they were lower in T1b (T0 vs T1b: $\mathrm{P}<0.05$; Tla vs T1b: $\mathrm{P}<0.05)$ and in $\mathrm{C}(\mathrm{T} 0$ vs $\mathrm{C}: \mathrm{P}<0.05$; T1a vs $\mathrm{C}$ : $\mathrm{P}<0.05$; T1b vs $\mathrm{C}: \mathrm{P}<0.05)$. Figure 2 summarizes graphically the main findings.

The inter-observer and intra-observer kappa statistics for semiquantitative measurements of immunohistochemical reactions showed values of 0.80 and 0.90 , respectively.

The correlation analysis between immunohistochemical levels of Hsps and inflammatory markers in lamina propria showed a linear correlation only between $\mathrm{Hsp} 90$ and CD4 levels in both T0 $(\mathrm{r}=+0.811 ; \mathrm{P}<0.005)$ and Tla $(\mathrm{r}=$ $+0.732 ; \mathrm{P}<0.005)$ groups (Figure 3 ).

\section{Discussion}

Ulcerative colitis (UC) affects the large bowel, particularly rectum and colon, in which it presents characteristic ulcers. ${ }^{12}$ In the intestinal mucosa, the lamina propria (LP) is infiltrated by abundant inflammatory cells, while the epithelium (Ep) undergoes cycles of destruction and repair by regeneration of basal cells. ${ }^{16}$

Although it is not universally accepted, UC is believed to have an autoimmune origin. ${ }^{17}$ Patients may develop systemic comorbidities and complications beyond the colon. These include aphthous ulcers of the mouth, ${ }^{18}$ uveitis, ${ }^{19}$ arthritis,${ }^{20}$ erythema nodosum, ${ }^{21}$ pyo-

Table 1. Semiquantitative evaluation of Hsp levels in the intestinal mucosa of patients with ulcerative colitis and controls with non-specific colitis*.

\begin{tabular}{lccccc} 
Hsp & Mucosal layer & T0 & Tla & T1b & C \\
Hsp10 & Ep & +++ & ++ & + & + \\
& LP & +++ & ++ & + & + \\
Hsp70 & Ep & +++ & ++ & + & + \\
& LP & + & + & + & + \\
\hline Hsp90 & Ep & +++ & +++ & +++ & + \\
& LP & ++ & ++ & + & + \\
\hline
\end{tabular}

*The immunohistochemical results are indicated in a semiquantitative scale (-: 0\%; +: 1-33\%; ++: 34-66\%; +++: 67-100\%); ${ }^{\circ} \mathrm{T} 0$, Tla, T1b, and C, mucosa taken: at time of diagnosis, after 6 months of treatment with 5-ASA, after 6 months of treatment with 5-ASA + probiotics, and from patients with non-pecific colitis (controls), respectively; Ep, epithelium; LP, lamina propria. 


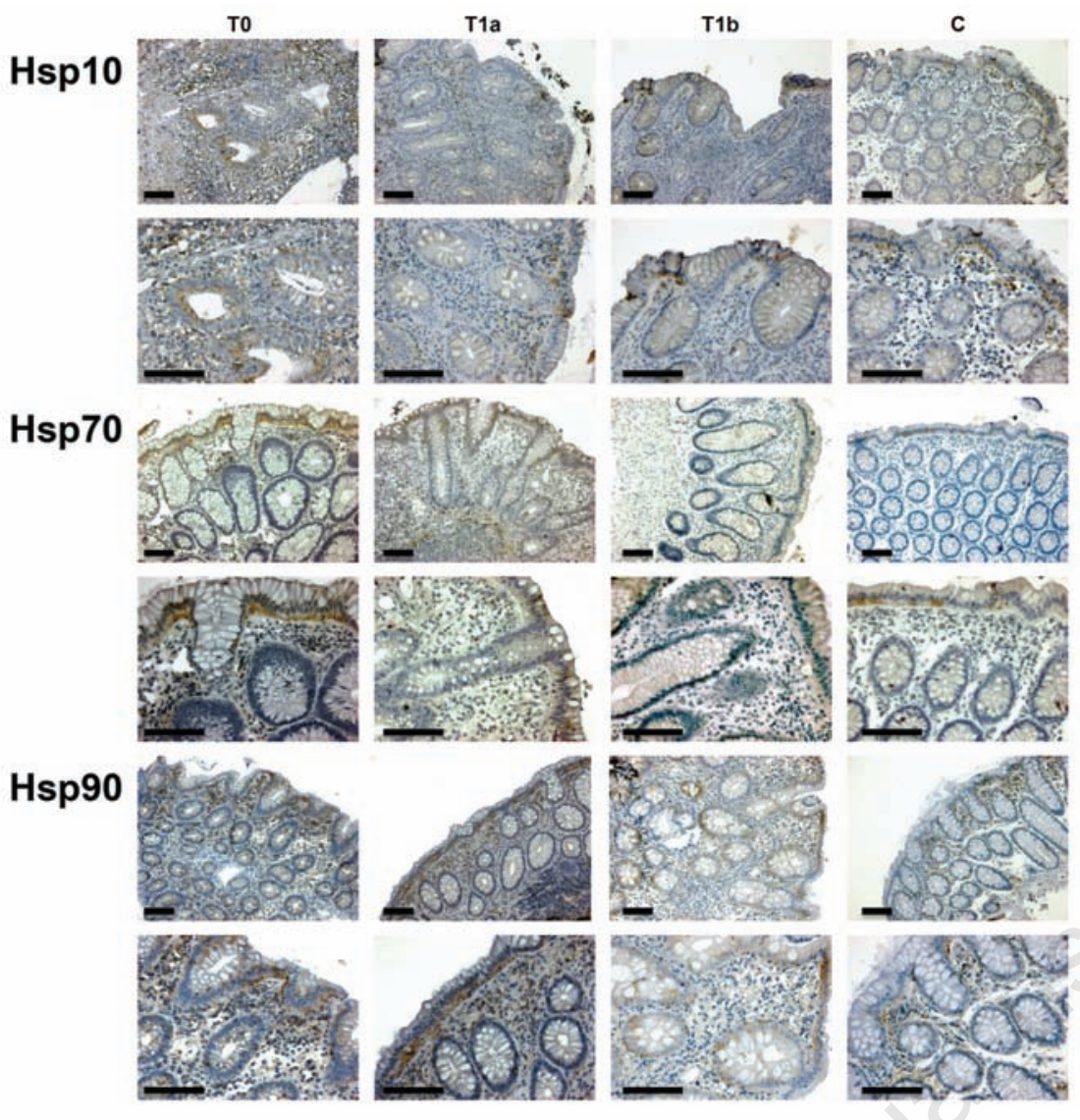

Figure 1. Representative pictures of immunohistochemical results. This figure shows microphotographs of immunostaining for $\mathrm{Hsp10}$, Hsp70, and $\mathrm{Hsp} 90$ in all groups studied T0, T1a, T1b, and C, with mucosa sampled: at time of diagnosis, after 6 months of treatment with 5-ASA, after 6 months of treatment with 5-ASA + probiotics, and from patients with non-specific colitis (controls), respectively. Positivity appears brown. Scale bars: $200 \mu \mathrm{m}$.

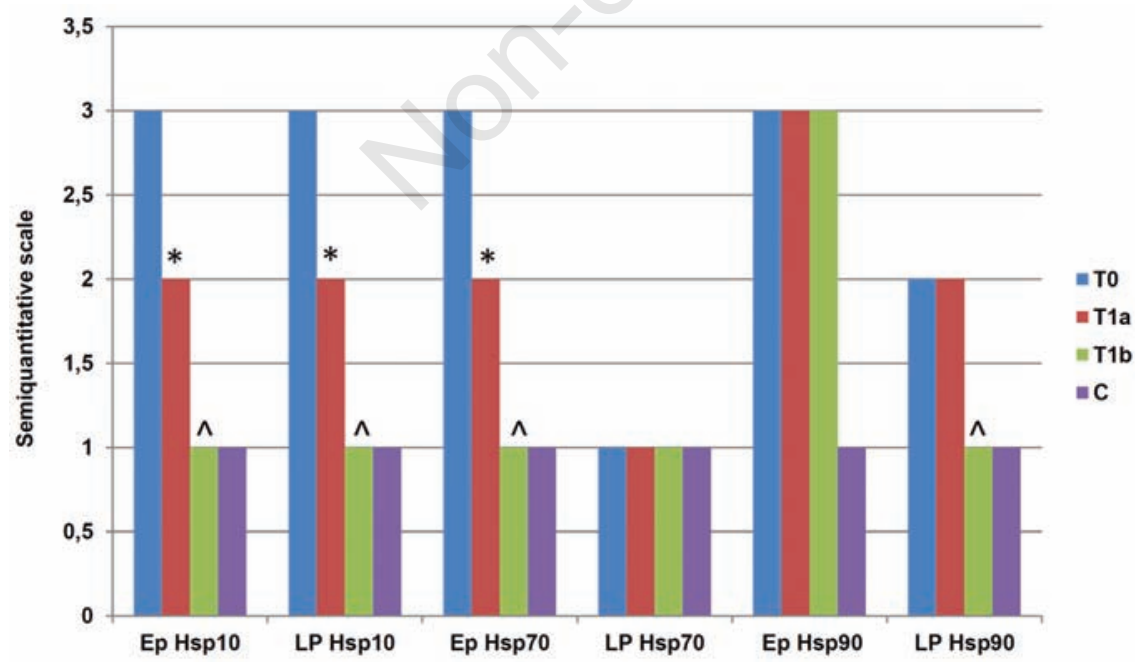

Figure 2. Graphic summary of the main findings. Statistical analyses of immunohistochemical data showed significant differences between T0 and T1 a $\left(^{*}\right)$ and between T0/T1a and $\mathrm{T} 1 \mathrm{~b}(\wedge)$ in epithelium (Ep) for Hsp10 and Hsp70 and in lamina propria (LP) for Hsp10 and Hsp90. Vertical axis: semiquantitative scale, as explained in the first footnote for Table 1 . derma gangrenosum, ${ }^{21}$ hemolytic anemia, ${ }^{22}$ and autoimmune hepatitis. ${ }^{23}$

Many of these autoimmune comorbidities have already been shown to be accompanied by increased levels of Hsps. For example, Hsp70 and Hsp90 have been found to increase in autoimmune arthritis, ${ }^{24,25} \mathrm{Hsp} 70$ was implicated in the development of autoimmune hepatitis; ${ }^{26}$ and levels of Hsp10, Hsp70, and Hsp90, as well as Hsp60, have been found to change in parallel with the development of large bowel cancer, ${ }^{27-29}$ the most severe complication of UC.

Hsp10 is classically considered the Hsp60 co-chaperonin, with both working inside mitochondria for assisting the correct folding of proteins. ${ }^{30}$ However, Hsp10 can also be found in extramitochondrial sites as well as in the extracellular environment, ${ }^{31}$ although the mechanism(s) responsible of its secretion has not yet been elucidated. Extracellular Hsp10 seems to have immunomodulatory activity, favoring the implant of blastocyst, ${ }^{32}$ as well as tumor progression. ${ }^{33}$

Hsp70 and Hsp90 are two of the most studied members of the Hsp family. ${ }^{34,35}$ They both have chaperone functions inside cells forming cytosolic chaperoning machines. ${ }^{34}$ In addition, they occur also in extracellular sites triggering immune system reactions, thus having proinflammatory effects. ${ }^{36,37}$
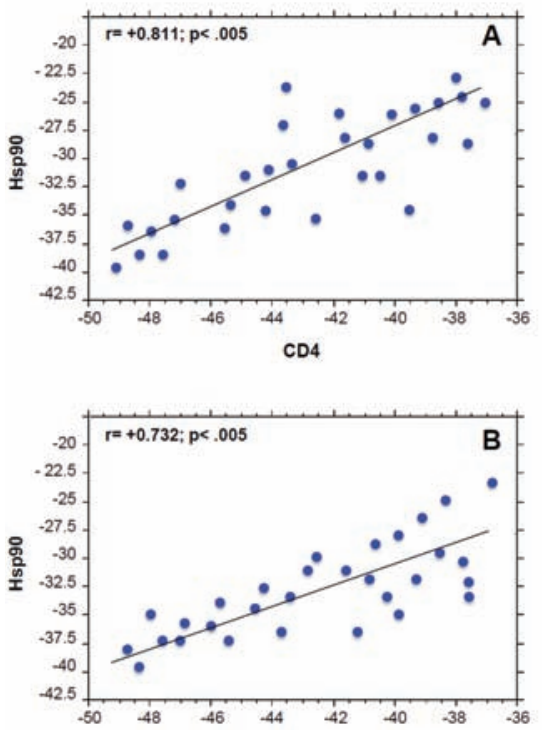

Figure 3. Positive correlation between Hsp90 and CD4 levels in Groups T0 (A) and T1a (B). Linear regression analysis between the levels of Hsp10, Hsp70, and Hsp90 with the levels of inflammatory markers (CD3, CD4, CD8, CD20, and CD68) demonstrated a positive correlation only between $\mathrm{Hsp} 90$ and CD4 only in Groups T0 and T1 a, as shown in the figure. The levels of the inflammatory markers were determined in a previous work. ${ }^{13}$ 
In this work, we found that the three Hsps we studied were elevated in the intestinal mucosal of all patients at the time of diagnosis. We also found that the Hsp levels changed significantly after therapy. For example, Hsp10 decreased in both the Ep and the LP in the Tla and T1b groups; Hsp70 decreased in T1a and T1b but only in the Ep; and Hsp90 decreased only in T1b and only in the LP.

Very little information exists on Hsp10 in $\mathrm{UC}$ and on the role this chaperonin might play in diagnostics as biomarker and in disease as a pathogenetic factor. In a previous work, we demonstrated that Hsp10 levels are increased in the intestinal mucosa of both CD and UC, compared to normal mucosa, and that it often co-localizes with Hsp60 in the same cells, both in Ep and LP. ${ }^{12}$ The results presented here show that Hsp10 levels in UC mucosa decrease after therapy. This decline is similar to that we previously described for Hsp60 after therapy. ${ }^{13}$ However, in contrast to Hsp60, Hsp10 has been described as an anti-inflammatory agent. Indeed, some clinical trials have already been performed demonstrating the usefulness of this protein in reducing inflammation in some autoimmune processes, as multiple sclerosis, ${ }^{38}$ severe plaque psoriasis, ${ }^{39}$ and rheumatoid arthritis..$^{40}$ Hence, we can postulate that the Hsp10 increase in UC at the time of diagnosis reflects an attempt of the mucosa to reduce the inflammation that is destroying the tissue; after therapy, when inflammation declines, Hsp10 levels in mucosal cells concomitantly decrease. By contrast, it seems that Hsp70 does not participate in the inflammation of the UC mucosa, since its levels in LP are commonly low and they did not present any change in the Groups we studied. Hsp70 could be considered a protective molecule for Ep cells. For instance, mice mutants for Hsp70, treated with a combination of chemical compounds that promote colitis and colon carcinogenesis, developed histological and molecular features of UC and cancer, while wild-type animals did not become sick. ${ }^{41}$ All together, these results are in agreement with previous studies that postulated a protective role of Hsp70 in UC. ${ }^{42}$ However, in another study Hsp70 could not be detected immunohistochemically in mucosa from UC patients. ${ }^{43}$

In an earlier work, Hsp90 was detected by immunohistochemistry in Ep and LP but no differences could be found in the intensity of staining or localization in patients with IBD compared to controls. ${ }^{44}$ By contrast, in our work reported here Hsp90 levels were higher in UC mucosa than in the mucosa of non-specific colitis and this could explain the discrepancy between our studies and those that used normal mucosa as base-line control. In addi- tion, we did not detect any changes in Hsp90 Ep levels after therapy. By contrast, treatment with 5-ASA + probiotics reduced Hsp90 levels in LP, while treatment with 5-ASA alone did not have any effect. In this regard, we found a linear correlation between Hsp90 and CD4 levels in LP of both T0 and Tla groups.

Hsp90 from the LP could reach the bloodstream and thus interact with the immune system since it has been reported that the chaperone is involved in activation of cells pertaining to innate and adaptive immunities, thereby affecting inflammation and autoimmunity. ${ }^{26,45}$ Based on results obtained with an experimental model using rodents, it has been proposed that a synthetic Hsp90 inhibitor, able to block LPS-induced TLR4 signaling of $\mathrm{CD} 4{ }^{+}$cells, could be applicable to treatment of autoimmune diseases involving inflammation and activation of the adaptive immune response. ${ }^{26}$ The data were in agreement with a previous work in which another Hsp90 inhibitor was found to reduce inflammation, cartilage damage, and bone resorption in a model of rheumatoid arthritis. ${ }^{45}$ Thus, we cannot exclude that increased levels of Hsp90 in LP of patients with UC could be followed by an inflammatory reaction triggered via $\mathrm{CD} 4^{+}$cells or by invasion of blood by the chaperone leading to autoimmune phenomena in extra-intestinal sites. We did not have blood samples from the patients investigated in the study reported here and, therefore, we could not measure Hsp90 in the sera of these patients, which would have allowed us to correlate chaperone levels in mucosa and in sera with the occurrence of comorbidities. If a positive correlation is found, one can then think that treatment of UC with 5-ASA plus probiotics should include attempts to reduce Hsp90 levels. The results of our correlation analysis between Hsp90 and CD4 levels in LP support this view.

In conclusion, Hsp10, Hsp70, and Hsp90 levels were increased in the mucosa of UC patients at the time of diagnosis, compared to non-specific colitis. These levels decreased after therapy. Combined treatment with 5-ASA and probiotics produced the greatest reduction in the Hsp levels, namely, the same pattern we had already found for Hsp60. ${ }^{13}$ This result is largely in agreement with other studies assessing the value of probiotics in treatment of IBD like UC..$^{46,47}$ All together, our results indicate that determination of Hsp levels in intestinal mucosa as done in this study has a promising potential for monitoring response to treatment in UC, and open new avenues for investigating the pathogenetic mechanisms of this disease, particularly in what concerns the role of the chaperones in the initiation and progression of the intestinal lesions.

\section{References}

1. Macario AJL, Lange M, Ahring BK, Conway de Macario E. Stress genes and proteins in the archaea. Microbiol Mol Biol Rev 1999;63:923-67.

2. Kampinga HH. Chaperones in preventing protein denaturation in living cells and protecting against cellular stress. Handb Exp Pharmacol 2006;172:1-42.

3. Prohászka Z, Füst G. Immunological aspects of heat-shock proteins-the optimum stress of life. Mol Immunol 2004;41: 29-44.

4. Cappello F, Conway de Macario E, Di Felice V, Zummo G, Macario AJL. Chlamydia trachomatis infection and anti-Hsp60 immunity: the two sides of the coin. PLoS Pathog 2009;5:e1000552.

5. Wick G, Knoflach M, Kind M, Henderson B, Bernhard D. Heat shock proteins and stress in atherosclerosis. Autoimmun Rev 2004;3:S30-1.

6. Lai Y, Chen C, Linn T. Innate immunity and heat shock response in islet transplantation. Clin Exp Immunol 2009;157:1-8.

7. Huang MN, Yu H, Moudgil KD. The involvement of heat-shock proteins in the pathogenesis of autoimmune arthritis: a critical appraisal. Semin Arthritis Rheum 2010;40:164-75.

8. Cappello F, Marino Gammazza A, Zummo L, Conway de Macario E, Macario AJL. Hsp60 and AChR cross-reactivity in myasthenia gravis: An update. J Neurol Sci 2010;292:117-8.

9. Otaka M, Odashima M, Watanabe S. Role of heat shock proteins (molecular chaperones) in intestinal mucosal protection. Biochem Biophys Res Commun 2006; 348:1-5.

10. Szigethy E, McLafferty L, Goyal A. Inflammatory bowel disease. Child Adolesc Psychiatr Clin N Am 2010;19:301-18.

11. Kaser A, Blumberg RS. Autophagy, microbial sensing, endoplasmic reticulum stress, and epithelial function in inflammatory bowel disease. Gastroenterology 2011;140:1738-47.

12. Rodolico V, Tomasello G, Zerilli M, Martorana A, Pitruzzella A, Marino Gam mazza A, et al. Hsp60 and Hsp10 increase in colon mucosa of Crohn's disease and ulcerative colitis. Cell Stress Chaperones 2010;15:877-84.

13. Tomasello G, Rodolico V, Zerilli M, Martorana A, Bucchieri F, Pitruzzella A, et al. Changes in immunohistochemical levels and subcellular localization after therapy and correlation and colocalization with 
CD68 suggest a pathogenetic role of $\mathrm{Hsp60}$ in ulcerative colitis. Appl Immunohistochem Mol Morphol 2011; [Epub ahead of print].

14. Heuschen UA, Allemeyer EH, Hinz U, Autschbach F, Uehlein T, Herfarth C, et al. Diagnosing pouchitis: comparative validation of two scoring systems in routine follow-up. Dis Colon Rectum 2002;45:776-86.

15. Cappello F, Barnes L. Synovial sarcoma and malignant mesothelioma of the pleura: review, differential diagnosis and possible role of apoptosis. Pathology 2001;33:142-8.

16. Gramlich T, Petras RE. Pathology of inflammatory bowel disease. Semin Pediatr Surg 2007;16:154-63.

17. Flanagan P, Campbell BJ, Rhodes JM. Bacteria in the pathogenesis of inflammatory bowel disease. Biochem Soc Trans 2011;39:1067-72.

18. Miyamoto NT Jr, Borra RC, Abreu M, Weckx LL, Franco M. Immune-expression of HSP27 and IL-10 in recurrent aphthous ulceration. J Oral Pathol Med 2008;37:4627.

19. Freeman HJ. Pouchitis-associated iritis (uveitis) following total proctocolectomy and ileal pouch-to-anal anastomosis in ulcerative colitis. Can J Gastroenterol 2001;15:131-3.

20. Yanai H, Hanauer SB. Assessing response and loss of response to biological therapies in IBD. Am J Gastroenterol 2011;106:68598.

21. Polcz M, Gu J, Florin T. Pyoderma gangrenosum in inflammatory bowel disease: the experience at Mater Health Services Adult Hospital 1998-2009. J Crohns Colitis 2011;5:148-51.

22. Leo Carnerero E, Aoufi S, Montero Cuadrado I, Herrera Martin P, Herrera Justiniano JM. Autoimmune hemolytic anemia associated with ulcerative colitis: response to infliximab. Am J Gastroenterol 2009;104:2370-1.

23. Saich R, Chapman R. Primary sclerosing cholangitis, autoimmune hepatitis and overlap syndromes in inflammatory bowel disease. World J Gastroenterol 2008;14: 331-7.

24. Wieten $L$, van der Zee $R$, Spiering $R$, Wagenaar-Hilbers J, van Kooten P, Broere F, et al. A novel heat-shock protein coinducer boosts stress protein Hsp70 to activate $\mathrm{T}$ cell regulation of inflammation in autoimmune arthritis. Arthritis Rheum 2010;62:1026-35.

25. Conroy SE, Faulds GB, Williams W, Latchman DS, Isenberg DA. Detection of autoantibodies to the $90 \mathrm{kDa}$ heat shock protein in systemic lupus erythematosus and other autoimmune diseases. $\mathrm{Br} \mathrm{J}$ Rheumatol 1994;33:923-6.
26. Yun TJ, Harning EK, Giza K, Rabah D, Li P, Arndt JW, et al. EC144, a synthetic inhibitor of heat shock protein 90 , blocks innate and adaptive immune responses in models of inflammation and autoimmunity. J Immunol 2011;186:563-75.

27. Cappello F, Bellafiore M, Palma A, David S, Marcianò V, Bartolotta $\mathrm{T}$, et al. $60 \mathrm{KDa}$ chaperonin (HSP60) is over-expressed during colorectal carcinogenesis. Eur J Histochem 2003;47:105-10

28. Cappello F, David S, Peri G, Farina F, Conway de Macario E, Macario AJL, et al. Hsp60: molecular anatomy and role in colorectal cancer diagnosis and treatment. Front Biosci 2011;3:341-51.

29. Cappello F, Czarnecka AM, La Rocca G, Di Stefano A, Zummo G, Macario AJL. Hsp60 and $\mathrm{Hspl0}$ as antitumor molecular agents. Cancer Biol Ther 2007;6:487-9.

30. Czarnecka AM, Campanella C, Zummo G, Cappello F. Heat shock protein 10 and signal transduction: a "capsula eburnea" of carcinogenesis? Cell Stress Chaperones 2006;11:287-94

31. Corrao S, Campanella C, Anzalone R, Farina F, Zummo G, Conway de Macario E, et al. Human Hsp10 and Early Pregnancy Factor (EPF) and their relationship and involvement in cancer and immunity: current knowledge and perspectives. Life Sci 2010;86:145-52.

32. Athanasas-Platsis S, Corcoran CM, Kaye PL, Cavanagh AC, Morton H. Early pregnancy factor is required at two important stages of embryonic development in the mouse. Am J Reprod Immunol 2000;43: 223-33.

33. Akyol S, Gercel-Taylor C, Reynolds LC, Taylor DD. HSP-10 in ovarian cancer: expression and suppression of T-cell signaling. Gynecol Oncol 2006;101:481-6.

34. Macario AJL, Conway de Macario E. Sick chaperones, cellular stress, and disease. N Engl J Med 2005;353:1489-501.

35. Macario AJL, Brocchieri L, Shenoy AR, Conway de Macario E. Evolution of a protein-folding machine: genomic and evolutionary analyses reveal three lineages of the archaeal hsp70(dnaK) gene. J Mol Evol 2006;63:74-86.

36. Vega VL, Rodríguez-Silva M, Frey T, Gehrmann M, Diaz JC, Steinem C, et al. Hsp70 translocates into the plasma membrane after stress and is released into the extracellular environment in a membraneassociated form that activates macrophages. J Immunol 2008;180:4299307.

37. Oura J, Tamura Y, Kamiguchi K, Kutomi G, Sahara H, Torigoe T, et al. Extracellular heat shock protein 90 plays a role in translocating chaperoned antigen from endosome to proteasome for generating antigenic peptide to be cross-presented by dendritic cells. Int Immunol 2011;23:22337.

38. Broadley SA, Vanags D, Williams B, Johnson B, Feeney D, Griffiths L, et al. Results of a phase Ila clinical trial of an anti-inflammatory molecule, chaperonin 10, in multiple sclerosis. Mult Scler 2009;15:329-36.

39. Williams B, Vanags D, Hall S, McCormack C, Foley P, Weiss J, et al. Efficacy and safety of chaperonin 10 in patients with moderate to severe plaque psoriasis: evidence of utility beyond a single indication. Arch Dermatol 2008;144:683-5.

40. Vanags D, Williams B, Johnson B, Hall S, Nash P, Taylor A, et al. Therapeutic efficacy and safety of chaperonin 10 in patients with rheumatoid arthritis: a double-blind randomised trial. Lancet 2006;368:855-63.

41. Tao Y, Hart J, Lichtenstein L, Joseph LJ, Ciancio MJ, Hu S, et al. Inducible heat shock protein 70 prevents multifocal flat dysplastic lesions and invasive tumors in an inflammatory model of colon cancer. Carcinogenesis 2009;30:175-82.

42. Nam SY, Kim N, Kim JS, Lim SH, Jung HC, Song IS. Heat shock protein gene 70-2 polymorphism is differentially associated with the clinical phenotypes of ulcerative colitis and Crohn's disease. J Gastroenterol Hepatol 2007;22:1032-8.

43. Lu XP, Omar RA, Chang WW. Immunocytochemical expression of the $70 \mathrm{kD}$ heat shock protein in ischaemic bowel disease. J Pathol 1996;179:409-13.

44. Stahl M, Ludwig D, Fellermann K, Stange EF. Intestinal expression of human heat shock protein 90 in patients with Crohn's disease and ulcerative colitis. Dig Dis Sci 1998;43:1079-87.

45. Rice JW, Veal JM, Fadden RP, Barabasz AF, Partridge JM, Barta TE, et al. Small molecule inhibitors of Hsp90 potently affect inflammatory disease pathways and exhibit activity in models of rheumatoid arthritis. Arthritis Rheum 2008;58:3765-75.

46. Do VT, Baird BG, Kockler DR. Probiotics for maintaining remission of ulcerative colitis in adults. Ann Pharmacother 2010;44:56571 .

47. Haller D, Antoine JM, Bengmark S, Enck P, Rijkers GT, Lenoir-Wijnkoop I. Guidance for substantiating the evidence for beneficial effects of probiotics: probiotics in chronic inflammatory bowel disease and the functional disorder irritable bowel syndrome. J Nutr 2010;140:690S-7. 